\title{
Positron emission tomography: An additional prognostic tool in dilated cardiomyopathy?
}

\author{
Danilo Neglia, MD, PhD, FESC \\ ${ }^{a}$ Fondazione G. Monasterio CNR-Regione Toscana and CNR Institute of Clinical Physiology, Pisa, \\ Italy
}

Received Jul 24, 2015; accepted Jul 24, 2015

doi: 10.1007/s12350-015-0254-9

\section{See related article, pp. 758-767}

Dilated cardiomyopathy (DCM) represents the most common cardiomyopathy worldwide. It is a heart muscle disorder defined by the presence of dilated and poorly functioning left or both ventricles. To qualify as DCM, the extent of myocardial dysfunction should not be explained exclusively by abnormal loading conditions or ischemic heart disease. DCM can be primary (genetic, mixed or predominantly familial non-genetic, or acquired) or secondary (inflammatory, autoimmune, or thyrotoxic). Nevertheless, in the majority of cases, no definite cause is found. ${ }^{1,2}$

The prevalence of DCM in the general population is thought to be $\approx 40$ to 50 cases per 100,000 and the incidence is $6.95 / 100,000$ new cases a year, but this is a rough estimation since there is a growing evidence that the course of the illness is asymptomatic and difficult to recognize for a long period. ${ }^{3,4}$ Transplant-free survival has improved in the last three decades from $55 \%$ to $71 \%$ and $87 \%$ at 8 years ${ }^{5}$ thanks to early diagnosis, effective management, and evidence-based integrated therapeutic approach including multiple drug therapy, implantable defibrillators, and cardiac resynchronization (CRT) treatments. Despite this success, DCM remains in most patients, a progressive disease with large individual

Reprint requests: Danilo Neglia, MD, PhD, Fondazione CNR Regione Toscana G. Monasterio and CNR Institute of Clinical Physiology, Via G. Moruzzi 1, 56126 Pisa, Italy; dneglia@ifc.cnr.it; http:// www.ftgm.it, http://www.ifc.cnr.it

J Nucl Cardiol 2016;23:768-72.

$1071-3581 / \$ 34.00$

Copyright (C) 2015 American Society of Nuclear Cardiology. variability and a poor prognosis. Accordingly, there is a need for tools able to better stratify risk in the single patient, to target treatment and assess response.

\section{VENTRICULAR FUNCTION AND PROGNOSIS IN DCM}

Several non-imaging clinical, laboratory, and instrumental findings have prognostic significance in DCM patients. However, non-invasive cardiac imaging is central for both characterization of disease and assessment of prognosis. The documentation by echocardiography or cardiac magnetic resonance imaging (CMR) of a dilated left ventricle (LV) with severely reduced ejection fraction $(\mathrm{EF})$, a restrictive diastolic filling pattern, and severe mitral regurgitation are all important indicators of the severity of the disease and prognostic indicators. ${ }^{6}$ Moreover, a growing importance as a prognostic indicator has been demonstrated by LV myocardial tissue characterization by late gadolinium enhancement (LGE) CMR. ${ }^{7}$

The prognostic role of impaired right ventricular (RV) function in DCM is increasingly recognized. Although the exact prevalence is poorly defined, RV systolic dysfunction has been reported in as many as $65 \%$ of patients ${ }^{8}$ suggesting that DCM is frequently a biventricular disease. The prognostic impact of RV impairment has been recently highlighted in a large study using CMR which is now considered as the gold standard for RV assessment. ${ }^{9}$ In a prospective series of 250 consecutive DCM patients evaluated by CMR, RV systolic dysfunction, defined as $\mathrm{RVEF} \leq 45 \%$, was a significant independent predictor of the primary end point of all cause mortality and heart transplantation in a median follow-up period of 6.8 years (hazard ratio, 3.90; 95\% CI 2.16-7.04; $P<0.001)$. On LGE-CMR, LV midwall fibrosis was present in $28 \%$ of the cohort and was more prevalent in patients with RV systolic dysfunction, while no patient was observed to have fibrous replacement of the RV free wall. 


\section{MYOCARDIAL BLOOD FLOW AND METABOLISM IN DCM}

The most commonly used imaging risk stratifiers in DCM describe the severity of LV and RV functional impairment or of myocardial damage. The extent of ventricular dysfunction which impacts on the prognosis, however, is not only the result of primary impairment of contractile function, irreversible myocardial damage (such as fibrosis), or of hemodynamic loads but could also depend on other conditions which characterize the individual myocardial substrate. Among other mechanisms, variation in myocardial perfusion and metabolism may play a relevant role in causing progressive ventricular impairment and adverse prognosis. Coronary microvascular dysfunction ${ }^{10}$ and myocardial metabolic shift toward preferential glucose utilization ${ }^{11}$ are some of the recognized underlying pathophysiologic mechanisms. It is expected that the final outcome as well as the possible response to treatment will depend on the interaction of interventions with this complex individual functional myocardial substrate.

Positron emission tomography (PET) allows an integrated qualitative and quantitative evaluation of myocardial perfusion and glucose uptake. The absolute levels of myocardial blood flow (MBF), measured by PET at rest and during stress, in the absence of obstructive coronary disease may express the coronary microvascular function. Using ${ }^{13} \mathrm{~N}$-Ammonia as a flow tracer, regional and global reduction of $\mathrm{MBF}$ can be demonstrated in up to $82 \%$ of patients with early stage $\mathrm{DCM}^{12}$ predicting the evolution toward progressive ventricular dysfunction and heart failure. ${ }^{13}$ Myocardial 18-Fluorodeoxyglucose ( ${ }^{18} \mathrm{~F}$-FDG) uptake, either qualitatively or quantitatively estimated by PET, describes the first step of myocardial glucose metabolism, since the tracer is actively transported and trapped into the myocardium not undergoing further metabolization. Using ${ }^{18}$ F-FDG PET, evidence of increased myocardial glucose uptake in regions with particularly depressed $\mathrm{MBF}$ can be frequently documented in DCM patients resembling the "mismatch" pattern characteristic of myocardial ischemia and hibernation in coronary artery disease. " This "mismatch", pattern is related with adverse outcome in terms of mortality, heart transplantation, and hospitalization ${ }^{15}$ suggesting that an "ischemic", myocardial metabolism may occur in DCM and contribute to progressive myocardial dysfunction and adverse outcome.

The cardiomyopathic heart may shift its metabolism toward higher utilization of glucose even independently from ischemia. There is direct evidence for a global chronic cardiac metabolic shift in DCM patients characterized by decreased free fatty acids (FFA) uptake and oxidation and increased carbohydrate utilization at rest as documented by measuring transmyocardial arteriovenous differences of oxygen and major metabolites. ${ }^{11}$ Non-invasive PET studies in non-ischemic cardiomyopathy found similar results. ${ }^{16,17}$ It is hypothesized that the shift in myocardial substrate utilization, partially resembling the neonatal metabolic phenotype, ${ }^{18}$ is a compensatory response of the severely impaired and energy-depleted myocardium which optimizes energy metabolism, by using glucose as a more efficient fuel. Myocardial metabolic changes at rest could be detrimental under stress conditions. At variance with normal hearts which shift their metabolism from preferential use of FFA to glucose under stress, cardiomyopathic hearts cannot further increase glucose uptake ${ }^{11}$ and rely upon FFA utilization which is associated with a lower myocardial mechanical efficiency. This mechanism can involve not only the whole myocardium but also specific regions. In DCM patients with left bundle branch block (LBBB), the highly coordinated and vigorously contracting lateral region tends to become thicker, and its metabolism shifts to near-maximal glucose utilization, becoming strongly dependent on this substrate. ${ }^{19}$ Such metabolic changes may further promote adverse cardiac remodeling that ultimately cannot be attenuated or eventually reversed by CRT.

\section{RV MYOCARDIAL BLOOD FLOW AND METABOLISM IN DCM}

Very few data are available on RV MBF and metabolism in DCM. This is mainly due to methodological difficulties. During heart catheterization, it is not possible to sample venous blood drained specifically from the RV. Information that can be gained by PET on perfusion and glucose metabolism of the LV myocardium is more difficult to be extended to the RV. The normal RV has a thickness which is comparable with the spatial resolution of most PET scanners posing the problem of partial volume effect which causes an artificial decrease in detected activity. Mielniczuk et al. ${ }^{20}$ have recently used ${ }^{18}$ F-FDG PET scans to estimate glucose metabolism in the right ventricle and the relation with $\mathrm{RV}$ function in patients with heart failure (HF). They studied 68 patients with advanced HF of non-ischemic or ischemic origin. Most of the patients had LBBB $(71 \%)$, diabetes was present in $45 \%$, and some patients had pulmonary hypertension. ${ }^{18} \mathrm{~F}-\mathrm{FDG}$ was injected after an oral glucose load or during euglycemic-hyperinsulinemic clamp in patients with diabetes. RV and LV FDG uptake were measured as standardized uptake value (SUV). Relative RV FDG uptake was determined as the ratio of RVSUV to 
LVSUV without or with correction for partial volume effect (wall thickness estimated by echocardiography). $R V{ }^{18}$ F-FDG uptake was weakly related to increased RV systolic pressure and not related to LV size, function, or ${ }^{18}$ F-FDG uptake. However, the authors found an inverse correlation of RVSUV/LVSUV ${ }^{18}$ F-FDG uptake with RVEF. They concluded that, in patients with HF, RV dysfunction is associated with an increase in $\mathrm{RV}{ }^{18} \mathrm{~F}$ FDG uptake relative to $\mathrm{LV}$, the magnitude of which is correlated with the severity of RV impairment.

In this issue of the journal, Wang et al used a similar approach to evaluate RV glucose metabolism in patients with non-ischemic DCM. ${ }^{21}$ CMR and ${ }^{18}$ F-FDG PET/CT imaging after oral glucose load were performed in 63 consecutive patients. RVSUV and LVSUV ${ }^{18} \mathrm{~F}-\mathrm{FDG}$ uptake on the PET images were corrected for partial volume effect by use of wall thickness measurements obtained by cardiac MRI. The authors confirmed a significant inverse correlation between corrected RV SUV (cRVSUV) values and RVEF $(r=-0.571$, $P<.001)$, as well as cRVSUV/cLVSUV values and $\operatorname{RVEF}(r=-0.405, P<.001)$. Over a median period of follow-up of 804 days, 15 of the patients $(23.8 \%)$ died or underwent cardiac transplant, the combined primary end point of the study. Corrected RVSUV values $>7.01$ and cRVSUV/cLVSUV values $>0.795$ were associated with hazard ratios for clinical end point of 5.4 and 6.4, respectively, on univariate analysis. On multivariate analysis, cRVSUV/cLVSUV values $>0.795$ remained a significant, independent predictor of the end point, with a hazard ratio of 5.0. The study of Wang et al. demonstrates that increased RV glucose metabolism is a common feature in patients with idiopathic DCM and confirms the relationship of RV ${ }^{18} \mathrm{~F}$-FDG uptake with the severity of RV dysfunction already documented in HF. Importantly, this study extends previous results by demonstrating the adverse prognostic role of relative increase of glucose metabolism in the RV as compared with the LV underlying the importance of biventricular functional and metabolic changes in these patients. Notably, relative increase of RV glucose metabolism outperformed RVEF as a prognostic indicator in the multivariate analysis. Metabolic changes occurring since the earliest stages of DCM in response to different disease mechanisms could be a more sensitive marker of an impaired myocardial substrate predisposing to progressive deterioration than measurements of ventricular dysfunction alone. In more advanced stages of the disease, the metabolic pattern could add more information than depressed RVEF.

The increased RV ${ }^{18} \mathrm{~F}$-FDG uptake may be part of the cardiomyopathic process being related to the substrate shift hypothesis. Myocardial metabolic shift to a more efficient fuel, expressing the adaptation of the heart muscle to conditions of lower oxygen availability due to coronary microvascular dysfunction and/or to increased wall stress could apply to both heart chambers. This metabolic alteration has been associated with an increased expression of glucose metabolism-related genes and proteins. ${ }^{22,23}$ Alternatively, it can be hypothesized that the relative increase in RV glucose metabolism may represent an adaptive or a maladaptive response of the RV myocardial metabolism to increased workload secondary to LV dysfunction. Insights into RV metabolism changes in conditions of RV overload can be obtained by studies in pulmonary arterial hypertension $(\mathrm{PAH})$. There is individual variation among $\mathrm{PAH}$ patients in their tendency to develop right heart failure. Some patients maintain a form of adaptive RV hypertrophy characterized by preservation of cardiac output, ejection fraction, and exercise capacity, while other patients develop maladaptive RV hypertrophy that refers to pathologic remodeling of the RV characterized by myocardial apoptosis, fibrosis, and severe dilatation and dysfunction. Recent evidence suggests that the variability in response to RV afterload may be due to variation in energy substrate metabolism. ${ }^{24}$ Limited PET studies, including one previous study by Wang et al., are available in PAH patients. ${ }^{25,26}$ These studies demonstrated that the magnitude of RV glucose uptake was associated with pressure overload and RV dysfunction using ${ }^{18}$ F-FDG PET. In a more recent study, Ohira et al. ${ }^{27}$ showed that the ratio of RV glucose over fatty acid uptake was related with pulmonary artery pressures and worsening of RV function in PAH. They also showed a frequent RV perfusion-FDG mismatch pattern in these patients suggesting the presence of RV hibernation and ischaemia driving the metabolic shift toward glycolysis. As a matter of fact, in PAH, as in DCM, an increase in RV glucose metabolism seems to be associated with a worse prognosis. Tatebe et al. ${ }^{28}$ recently first reported that enhanced RV free wall SUV of ${ }^{18} \mathrm{~F}$ FDG is a predictor of clinical worsening and mortality in these patients.

\section{CLINICAL IMPLICATIONS}

The study of Wang et al. underlines the usefulness of using PET to characterize the myocardial functional substrate and to further stratify prognosis in patients with DCM besides common evaluation of biventricular cardiac impairment and myocardial damage as can be obtained by other methods. The combined assessment of myocardial blood flow and metabolism is able to disclose the presence and extent of myocardial functional abnormalities due to microvascular ischemia and/ or metabolic adaptation to increased hemodynamic loads. The feasibility to image by PET the RV together 
with the LV allows to get information on biventricular involvement in the disease process. Further studies would be needed to document whether earlier recognition of this altered biventricular substrate could help to better select responders to treatment including cardiac resynchronization and metabolic modulators ${ }^{29}$ or should be used to early address patients to cardiac transplantation. The availability of hybrid PET/MR scanners ${ }^{30}$ could allow in the future an even more comprehensive evaluation of DCM patients adding together multiple prognostic indicators spanning from biventricular function, to myocardial damage analysis to qualitative and possibly quantitative evaluation of biventricular flow and metabolism. The usefulness of such approach should be evaluated in terms of documented benefits against increased costs.

\section{Disclosure} disclose.

The authors have no conflict of interest nothing to

\section{References}

1. Maron BJ, Towbin JA, Thiene G, Antzelevitch C, Corrado D, Arnett D, Moss AJ, Seidman CE, Young JB. Contemporary definitions and classification of the cardiomyopathies: An American Heart Association Scientific Statement from the Council on Clinical Cardiology, Heart Failure and Transplantation Committee; Quality of Care and Outcomes Research and Functional Genomics and Translational Biology Interdisciplinary Working Groups; and Council on Epidemiology and Prevention. Circulation 2006;113:1807-16.

2. Elliott P, Andersson B, Arbustini E, Bilinska Z, Cecchi F, Charron P, Dubourg O, et al. Classification of the cardiomyopathies: A position statement from the European Society Of Cardiology Working Group on Myocardial and Pericardial Diseases. Eur Heart J 2008;29:270-6.

3. Rosamond W, Flegal K, Furie K, Go A, Greenlund K, Haase N, et al. Heart disease and stroke statistics-2008 update: A report from the American Heart Association Statistics Committee and Stroke Statistics Subcommittee. Circulation 2008;117:e25-146.

4. Redfield MM, Gersh BJ, Bailey KL, Ballard DJ, Rodeheffer RJ. Natural history of incidentally discovered, asymptomatic idiopathic dilated cardiomyopathy. Am J Cardiol 1994;74:737-9.

5. Merlo M, Pivetta A, Pinamonti B, Stolfo D, Zecchin M, Barbati G, et al. Long-term prognostic impact of therapeutic strategies in patients with idiopathic dilated cardiomyopathy: Changing mortality over the last 30 years. Eur J Heart Fail 2014;16:317-24.

6. Bart BA, Shaw LK, McCants CB Jr, Fortin DF, Lee KL, Califf $\mathrm{RM}$, et al. Clinical determination of mortality in patients with angiographically diagnosed ischemic or nonsichemic cardiomyopathy. J Am Coll Cardiol 1997;30:1002-8.

7. Kuruvilla S, Adenaw N, Katwal AB, Lipinski MJ, Kramer CM, Salerno M. Late gadolinium enhancement on cardiac magnetic resonance predicts adverse cardiovascular outcomes in nonischemic cardiomyopathy: A systematic review and meta-analysis. Circ Cardiovasc Imaging 2014;7:250-8.
8. La Vecchia L, Zanolla L, Varotto L, Bonanno C, Spadaro GL, Ometto R, et al. Reduced right ventricular ejection fraction as a marker for idiopathic dilated cardiomyopathy compared with ischemic left ventricular dysfunction. Am Heart J 2001;142:181-9.

9. Gulati A, Ismail TF, Jabbour A, Alpendurada F, Guha K, Ismail NA, et al. The prevalence and prognostic significance of right ventricular systolic dysfunction in nonischemic dilated cardiomyopathy. Circulation 2013;128:1623-33.

10. Neglia D, L'Abbate A. Coronary microvascular dysfunction and idiopathic dilated cardiomyopathy. Pharmacol Rep 2005;57:151-5.

11. Neglia D, De Caterina A, Marraccini P, Natali A, Ciardetti M, Vecoli $\mathrm{C}$, et al. Impaired myocardial metabolic reserve and substrate selection flexibility during stress in patients with idiopathic dilated cardiomyopathy. Am J Physiol Heart Circ Physiol 2007;293:H3270-8

12. Neglia D, Parodi O, Gallopin M, Sambuceti G, Giorgetti A, Pratali L, et al. Myocardial blood flow responce to pacing tachycardia and to dipyradamole infusion in patients with dilated cardiomyopathy without overt heart failure. Circulation 1995;92:796-804.

13. Neglia D, Trivieri MG, Michelassi C, Gallopin M, Pratali L, Poddighe R, et al. Prognostic value of myocardial blood flow impairment in patients with idiopathic left ventricular dysfunction. Circulation 2002;105:186-93.

14. van den Heuvel AF, van Veldhuisen DJ, van der Wall EE, Blanksma PK, Siebalink HM, Vaalburg WM, et al. Regional myocardial blood flow reserve impairment and metabolic changes suggesting myocardial ischemia in patients with idiopathic dilated cardiomyopathy. J Am Coll Cardiol 2000;35:19-28.

15. de Jong RM, Tio RA, van der Harst P, Voors AA, Koning PM, Zeebregts $\mathrm{CJ}$, et al. Ischemic patterns assessed by positron emission tomography predict adverse outcome in patients with idiopathic dilated cardiomyopathy. J Nucl Cardiol 2009;16:76974.

16. Tuunanen H, Engblom E, Naum A, Scheinin M, Nagren K, Airaksinen J, et al. Decreased myocardial free fatty acid uptake in patients with idiopathic dilated cardiomyopathy: Evidence of relationship with insulin resistance and left ventricular dysfunction. J Card Fail 2006;12:644-52.

17. Davila-Roman VG, Vedala G, Herrero P, de las de las Fuentes L, Rogers JG, Kelly DP, et al. Altered myocardial fatty acid and glucose metabolism in idiopathic dilated cardiomyopathy. J Am Coll Cardiol 2002;40:271-7.

18. Stanley WC, Recchia FA, Lopaschuk GD. Myocardial substrate metabolism in the normal and failing heart. Physiol Rev 2005;85:1093-129.

19. Masci PG, Marinelli M, Piacenti M, Lorenzoni V, Positano V, Lombardi M, et al. Myocardial structural, perfusion, and metabolic correlates of left bundle branch block mechanical derangement in patients with dilated cardiomyopathy: A tagged cardiac magnetic resonance and positron emission tomography study. Circ Cardiovasc Imaging 2010;3:482-90.

20. Mielniczuk LM, Birnie D, Ziadi MC, deKemp RA, DaSilva JN, Burwash I, et al. Relation between right ventricular function and increased right ventricular [18F]fluorodeoxyglucose accumulation in patients with heart failure. Circ Cardiovasc Imaging 2011;4:5966.

21. Wang L, Ma X, Xiang L, Lu M, Yan C, Zhao S, Fang W. The characterization and prognostic significance of right ventricular glucose metabolism in non-ischemic dilated cardiomyopathy. $\mathbf{J}$ Nucl Cardiol 2015. doi:10.1007/s12350-015-0165-9

22. Ardehali H, Sabbah HN, Burke MA, Sarma S, Liu PP, Cleland JG, et al. Targeting myocardial substrate metabolism in heart failure: Potential for new therapies. Eur J Heart Fail 2012;14:120-9. 
23. von Lewinski D, Gasser R, Rainer PP, Huber MS, Wilhelm B, Roessl U, et al. Functional effects of glucose transporters in human ventricular myocardium. Eur J Heart Fail 2010;12:106-13.

24. Neubauer S. The failing heart-an engine out of fuel. N Engl J Med 2007;356:1140-51.

25. Bokhari S, Raina A, Rosenweig EB, Schulze PC, Bokhari J, Einstein AJ, et al. PET imaging may provide a novel biomarker and understanding of right ventricular dysfunction in patients with idiopathic pulmonary arterial hypertension. Circ Cardiovasc Imaging 2011;4:641-7.

26. Wang L, Zhang Y, Yan C, He J, Xiong C, Zhao S, et al. Evaluation of right ventricular volume and ejection fraction by gated $18 \mathrm{~F}$ FDG PET in patients with pulmonary hypertension: Comparison with cardiac MRI and CT. J Nucl Cardiol 2013;20:242-52.

27. Ohira H, deKemp R, Pena E, Davies RA, Stewart DJ, Chandy G, et al. Shifts in myocardial fatty acid and glucose metabolism in pulmonary arterial hypertension: A potential mechanism for a maladaptive right ventricular response. Eur Heart J Cardiovasc Imaging 2015. doi:10.1093/ehjci/jev136.

28. Tatebe S, Fukumoto Y, Oikawa-Wakayama M, Sugimura K, Satoh K, Miura Y, et al. Enhanced [18F]fluorodeoxyglucose accumulation in the right ventricular free wall predicts long-term prognosis of patients with pulmonary hypertension: A preliminary observational study. Eur Heart J Cardiovasc Imaging 2014;15:666-72.

29. Tuunanen H, Engblom E, Naum A, Någren K, Scheinin M, Hesse $\mathrm{B}$, et al. Trimetazidine, a metabolic modulator, has cardiac and extracardiac benefits in idiopathic dilated cardiomyopathy. Circulation 2008;118:1250-8.

30. Rischpler C, Nekolla SG, Dregely I, Schwaiger M. Hybrid PET/ MR imaging of the heart: Potential, initial experiences, and future prospects. J Nucl Med 2013;54:402-15. 